\title{
MODELLING AND DYNAMIC ANALYSIS OF THREE-DIRECTION GRID PRESTRESSED RETICULATED MEGA-STRUCTURE
}

\author{
Wenbo Zhang ${ }^{1}$, Fang Liu ${ }^{2}$, Liangliang Zou³ and Pei xiang Chen²
}

\author{
1. Beijing Municipal Engineering Consulting Corporation, Beijing, China; \\ WenboZhang@shu.edu.cn \\ 2. Anhui Civil Airport Group Co., Anhui, China; 2608836030@qq.com, \\ chenpx2012@163.com \\ 3.Wenzhou Longwan Internation Airport, Zhejiang; China; 1070965597@qq.com
}

\begin{abstract}
In this paper, the generation way of three-direction grid prestressed reticulated megastructure is studied, and the three-direction grid prestressed reticulated mega-structure composite joint specific formation ideas are proposed. The important control parameters of three-direction grid prestressed reticulated mega-structure are determined, and the geometric modelling method of hexagon across the middle joint and octagon across the side joint is proposed. Coordinate formula of hexagonal and octagonal joints are derived to ensure reasonable grid arrangement and uniform stress distribution of the structure. Combined with the geometric parameters of the mega grid, a new method for creating the geometric model of three-direction grid prestressed mega reticulated structure is realized. The quadrangular pyramid space grid structure with the same length, span, rise and grid size and the three-direction grid prestressed grid structure are calculated, and the advantages of three-direction grid prestressed grid structure in dynamic performance are compared and analyzed.
\end{abstract}

\section{KEYWORDS}

Three-direction grid, Prestress, Giant grid, Geometric model

\section{INTRODUCTION}

With the development of economy and the improvement of human needs, people have higher requirements for spatial structure, especially the structural span. However, as the span increases, some problems within the space structure appear, such as the excessively small thickness-to-span ratio caused by the overall instability of the structure, excessive internal force of the component, and instability caused by the excessively long component. In recent years, the mega lattice structure system and the prestressed lattice structure system [4-6] proposed by $\mathrm{He}$ Yongjun and other scholars [1-3] can better improve the spanning capacity of the structure. Compared with ordinary grid structure, the material utilization rate of giant grid structure is higher, and the material used per unit building area is less, so the self-weight of the structure is relatively small. The stress of the structure is clear, although the stress of the member is relatively large, the phenomenon of excessive stress of a single member is effectively avoided and the deflection of the 
structure is effectively reduced due to the existence of an assembly or a three-direction truss. However, the application of reticulated mega-structure in engineering has also some limitations. The stiffness of the reticulated mega-structure is usually smaller than that of the ordinary reticulated structure. In order to meet the design specifications, the reticulated mega-structure needs to be cambered, that is to form a reticulated mega structure with a certain rise to meet the structural stiffness requirements. The limitation of the giant network is more obvious in the case that the design is restricted by the height and the internal height is required, for example, in the design of the hangar roof structure, the height is restricted by the airport clearance, and directly adopting the giant reticulated shell structure as the hangar roof structure can cause the internal height of the hangar to be insufficient and influence the use function. In view of the above problems, it is particularly important to study a new type of large-span structure with small thickness and large stiffness.

Ding Leran [7] carried out the corresponding research on the giant grid suspen-dome structure with a span of $200 \mathrm{~m}$, and based on the analysis of static, stability and dynamic characteristics of the structure, the optimal range of geometric control parameters is determined. Hu Duo [8] carried out the corresponding research on the honeycomb grid spherical reticulated shell with a span of $100 \mathrm{~m}$, and comprehensively analyzed the geometric composition, internal force distribution law and main control parameters of the main structure, which effectively improved the theoretical guidance for the poor mechanical performance of the structure in practical engineering. Yao Li [9] made a systematic study on the super-long span Kiewitt reticulated megastructure with the span of $800 \mathrm{~m}$, and put forward a more reasonable forming method for the megareticulated structure by using CAD three-direction modeling analysis. He Yongjun [10-12] studied the mechanical properties and related parameters of the main structure of the cylindrical intersecting three-direction truss system, and made a detailed analysis and description on the composition of the main structure, the selection of body parameters and supporting methods, as well as the sub-structure and its connection with the main structure. In this paper, the threedirection grid type prestressed reticulated mega-structure is based on the grid structure as the basic structure, combined with the idea of the mega-grid structure, that is to retain the member on the main force path, remove the member on the secondary force path. Besides, in order to meet the structural stiffness, prestressed cables replace the bottom chord of the mega-component in the main force direction. Finally, a three-direction grid type prestress mega-structure is formed. The Dynamic performance is the important content of the analysis on the mechanical performance of the prestressed three-direction grid mega-structure. In order to compare the advantages of the prestressed three-direction grid structure, the quadrilateral pyramid space grid structure and prestressed three-direction grid mega-structure with the same length, span, rise and grid size are calculated. From the structure of the dynamic performance of the comparative analysis. 


\section{Composition of mega-member and establishment of composite joint}

Through the comprehensive consideration of the characteristics of several commonly used flat grid forms, the inverted pyramid truss is finally selected as the lattice mega component of the three-direction grid prestressed grid structure, and the inverted pyramid truss is specifically shown in Figure1.

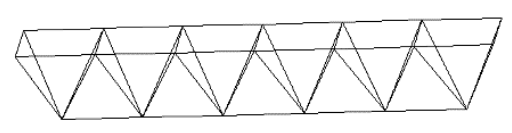

Fig. 1 - Inverted quadrangular pyramid truss

After the flat grid frame is evacuated, a three-direction grid type single-layer grid structure is formed, and the configuration is shown in Figure 2.

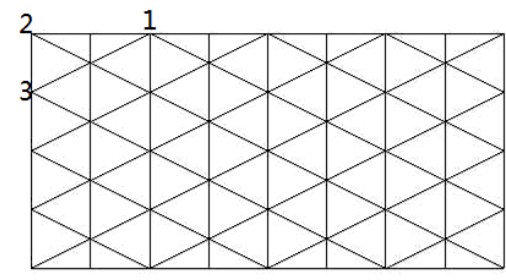

Fig. 2 - Evacuated Grid Structure

When a single bar is replaced by pyramid truss, the joint between mega components is complex. The joint of inverted pyramid truss structure is the key to form the geometric model of three-direction grid prestressed mega grid structure, which is called "composite joint" in this paper. Joints 1, 2 and 3 indicated in Figure 2 represent three types of connection forms respectively. Joint 1 with long direction indicates that five members are connected at the joint. Corner point 2 indicates that three members are connected at the joint. Joint 3 with cross direction indicates that four members are connected, indicating that the structure needs three different treatment methods to connect the inverted quadrangular pyramid trusses on the four edges. In order to make the modeling of the geometric model of the structure meet the connection of three different joints at the same time, eight groups of inverted quadrangular pyramid trusses are connected, and an inverted octagonal pyramid with an octagonal plane projection is formed at the cross connection. And that eight side on the upper part of the truss can simultaneously meet the form that the joints of the invert quadrangular pyramid truss are mutually crossed and connected in the three forms.

In addition to the four external giant components need to be connected to each other, the internal joints of the structure are connected to each other by six bars. Six groups of inverted quadrangular pyramid trusses are crosswise connected to form a composite joint with a hexagonal plane projection; Through the above treatment of the external and internal composite joints, a three-direction grid type prestressed mega-grid structure is finally formed as shown in Figure 3 , wherein $(A)$ is an axonometric view of the structure, and $(B)$ is a plan view of the structure. 


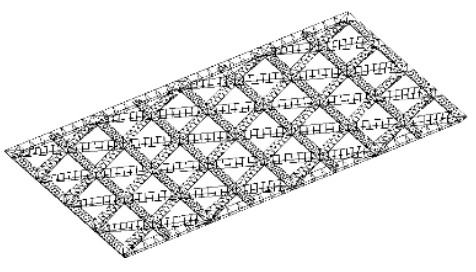

(A) Axonometric drawing of three-direction grid type prestressed giant grid structures

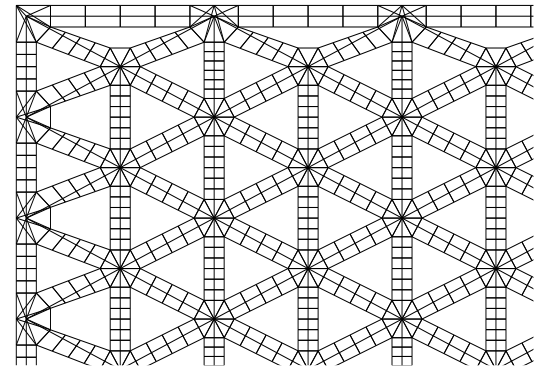

(B) Top view of prestressed mega-grid structure with three-direction grid

Fig. 3 - Schematic diagram of three-direction grid type prestressed mega-grid structures

\section{METHOD FOR FORMING GEOMETRIC MODEL OF THREE-DIRECTION GRID PRESTRESSED RETICULATED MEGA-STRUCTURE}

\section{Formation of Composite Joint}

The geometric model of three-direction grid prestressed reticulated mega-structure is essentially a flat plate-type space structure, so the establishment of the geometric model of the hexagonal mid-joint and the octagonal side joint is the key to generate the structural model. The above-mentioned "hexagon" refers to that projection of the inverted quadrangular pyramid truss in the interior of the structure on the intersect connecting plane, and the "octagon" refers to the projection of the inverted quadrangular pyramid truss on four out edges on the intersecting connecting plane. The specific connection mode is shown in Figure 4, wherein $(A)$ is the connection mode of the internal inverted quadrangular pyramid;(B) is an inverted quadrangular pyramid connection with four outer edges.

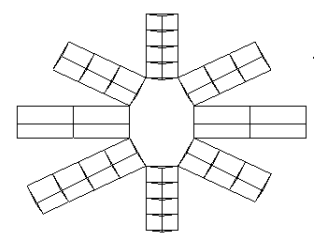
(A) Cross structure projected as hexagon
(B) Cross structure projected as octagon

Fig. 4- Hexagonal and Octagonal Connections

As shown in the figure above, both the internal and external connection modes of the cross connection part are hollowed out, which will cause uneven force on the bar and adversely affect the overall stiffness. And therefore that diagonal line of the upper polygons of the hollow structure need to be mutually crossed and connected; As the octagonal protruding structure part on four sides is easy to be stressed and concentrated, the part protruding out of the outer edge of the quadrangular pyramid is cut off. The corner points of the hexagon and the trimmed octagon are respectively connected with the corresponding joints of the three-direction grid type single-layer 
grid structure to form partial web members of the three-direction grid type prestressed mega-grid structure. Finally, a composite joint is formed as shown in Figure 5.

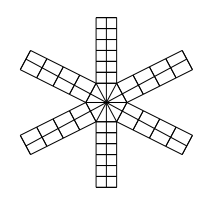

(A) Hexagonal internal connection (B) Octagonal internal connection

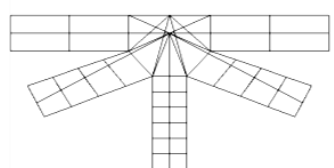

Fig. 5 - Composite joint in final form

\section{Geometric parameter setting of mega-structure}

According to the characteristics of the three-direction grid type prestress mega-grid structure, the geometric parameters of the mega-grid are set as shown in Figure 6, wherein Lx represents the length of the structural longitudinal mega-grid, Ly represents the length of the structural transversal mega-grid, and $\mathrm{H}$ represents the thickness of the structure; The number of long direction giant grids $\mathrm{Nx}$, the number of span direction giant grids $\mathrm{Ny}$, the number of long direction giant component interjoint grids $\mathrm{N} 1$, the numbers of span direction giant component interjoint grids N2, and the numbers of oblique direction giant component interjoint grids N3.

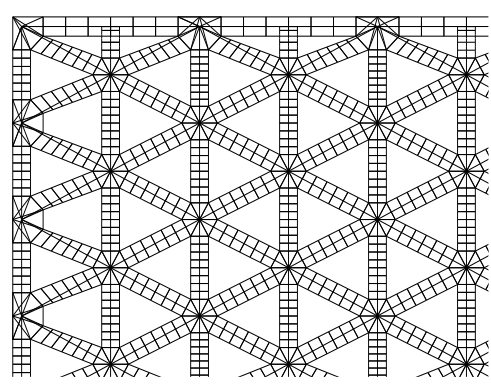

(A) Structural plan

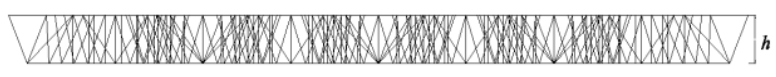

(B) Structural elevation

Fig. 6-Geometric parameters of structures

The projections of the midspan composite joint and the edge composite joint on the lower chord plane are hexagons and octagons respectively, and the hexagons and octagons are not necessarily regular polygons. The specific geometric parameters are shown in Figure 7 (a). 


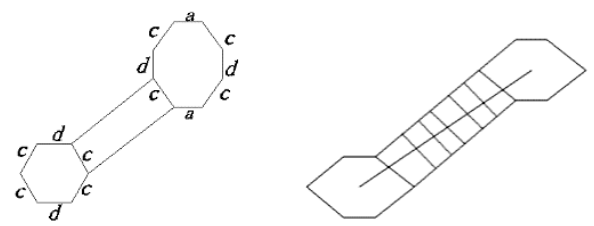

(a) Hexagonal and octagonal geometry (b) Non-uniform case of giant grid

Fig. 7 - Compound joints are not regular polygon

The single member of the three-direction grid single-layer reticulated structure is replaced by the inverted pyramid truss. In order to make the huge grid arrangement even, the overall structure stress reasonable, the member type reduces as far as possible, so the inverted quadrangular pyramid truss must be arranged reasonably. In the structure shown in Figure 8 , the lines connecting the central point of the hexagon to the four corner points are equal, that is, the lengths of the four line segments are equal and are all e; The connecting lines of the central point of the hexagon and the remaining two corner points are equal, and the lengths of the two line segments are B; In order to ensure that the members are evenly distributed and properly stressed, $\mathrm{I}$ in the illustration must be perpendicular and bisect $\mathrm{C}$.

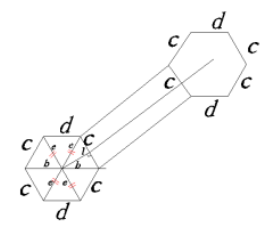

Fig. 8 - Diagram of the relationship between the hexagons

To sum up, in order to reduce the structural mega grid to the form as shown in Figure 7 (b), and ensure uniform bar specification and uniform stress, the triangle divided by the hexagon must be a regular triangle, that is, $b=e$, so the hexagon of the projection of the mid-span composite joint in the lower chord plane must be a regular hexagon.

Similarly, the octagon with side spans is also designed as a regular octagon, and the side length of the octagon is also $\mathrm{C}$. The number of the long direction giant component interjoint grids is $\mathrm{N} 1$, the number of the cross direction giant component interjoint grids is $\mathrm{N} 2$, and the number of the oblique direction giant component interjoint grids is N3, and the length of each corresponding small grid is respectively L1, L2 and L3, which are respectively expressed by formulas (1), (2) and (3). Figure 9 (a) Length L2 and L3 of that transversal and oblique small grids, wherein L3 is divide into two parts which are respectively positioned between two hexagons and between a hexagon and an octagon, and are respectively denote by L3 and L3; Figure 9 (b) is the length L1 of the lengthwise small mesh;

$$
l_{1}=\frac{L_{x}-\frac{c}{\tan 22.5^{\circ}}}{n_{1}} .
$$




$$
\begin{gathered}
l_{2}=\frac{L_{y}-\frac{\sqrt{3}}{2} c}{n_{2}} \\
l_{3}=\frac{\sqrt{\frac{L_{x}^{2}+L_{y}^{2}}{2}}-\frac{\sqrt{3}}{2} c-\frac{c}{2 \sin 22.5^{\circ}}}{n_{3}} \\
l_{3}=\frac{\sqrt{\frac{L_{x}^{2}+L_{y}^{2}}{2}-\sqrt{3} c}}{n_{3}}
\end{gathered}
$$
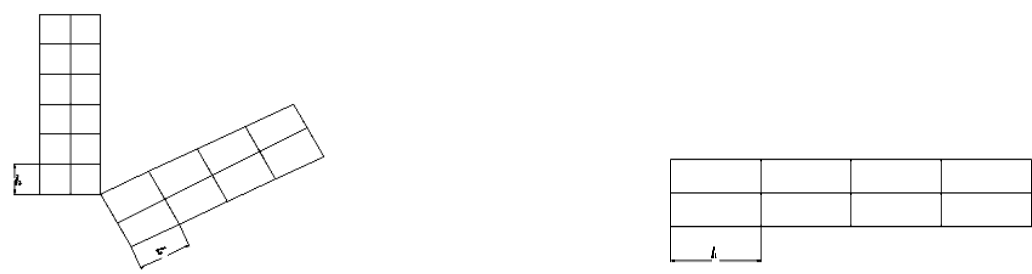

(a) Cross-direction and diagonal small grid length (b) Long-direction small grid length

Fig. 9 - Length of small grid

\section{Structural Geometric Parameter Scheme}

In order to accurately express the relationship between the coordinate value of each point and the length of the polygon, this paper establishes the local coordinate origin at the center point of the hexagon and octagon, and stipulates the serial number of the corner points of the hexagon and octagon as shown in Figure 10.

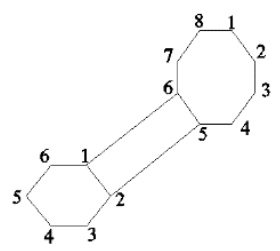

Fig. 10 - Sequence number of corner point of arbitrary polygon

According to the length of each side of the hexagon or the octagon, the coordinates of each corner point of the hexagon and the octagon in the local coordinate system can be expressed. The hexagon $S$ and the octagon $E$ can be expressed by the side length of the hexagon in local coordinates, and the coordinate formula of each corner point is $(X S j, Y S j)$, and the value range of $J$ is 1-6; The side length of the octagon represents that the coordinate formula of each corner point is (XEk, YEk), and the value range of $\mathrm{K}$ is $1-8$. The local coordinate formulas of the six corner points of the hexagon are shown in (4)- (9): 


$$
\begin{aligned}
& \left\{\begin{array}{l}
X_{S 1}=\frac{1}{2} c \\
Y_{S 1}=\frac{\sqrt{3}}{2} c
\end{array}\right. \\
& \left\{\begin{array}{l}
X_{S 2}=c \\
Y_{S 2}=0
\end{array}\right. \\
& \left\{\begin{array}{l}
X_{S 3}=\frac{\sqrt{3}}{2} c \\
Y_{S 3}=-\frac{1}{2} c
\end{array}\right. \\
& \left\{\begin{array}{l}
X_{S 4}=-\frac{\sqrt{3}}{2} c \\
Y_{S 4}=-\frac{1}{2} c
\end{array}\right. \\
& \left\{\begin{array}{l}
X_{S 5}=-c \\
Y_{S 5}=0
\end{array}\right. \\
& \left\{\begin{array}{l}
X_{S 6}=\frac{1}{2} c \\
Y_{S 6}=-\frac{\sqrt{3}}{2} c
\end{array}\right.
\end{aligned}
$$

The local coordinate formulas of the eight corner points of the octagon are shown in (10)(17):

$$
\begin{aligned}
& \left\{\begin{array}{l}
X_{E 1}=\frac{1}{2} c \\
Y_{E 2}=\frac{c}{2 \tan 22.5^{\circ}}
\end{array}\right. \\
& P=\left(N_{1}+N_{2}\right) \cos \theta \\
& \left\{\begin{array}{l}
X_{E 3}=\frac{c}{2 \tan 22.5^{\circ}} \\
Y_{E 3}=-\frac{c}{2}
\end{array}\right.
\end{aligned}
$$




$$
\begin{aligned}
& \left\{\begin{array}{l}
X_{E 4}=\frac{1}{2} c \\
Y_{E 4}=-\frac{c}{2 \tan 22.5^{\circ}}
\end{array}\right. \\
& \left\{\begin{array}{l}
X_{E 5}=-\frac{1}{2} c \\
Y_{E 5}=-\frac{c}{2 \tan 22.5^{\circ}}
\end{array}\right. \\
& \left\{\begin{array}{l}
X_{E 6}=-\frac{c}{2 \tan 22.5^{\circ}} \\
Y_{E 6}=-\frac{c}{2} \\
\left\{\begin{array}{l}
X_{E 7}=-\frac{c}{2 \tan 22.5^{\circ}} \\
Y_{E 7}=\frac{c}{2} \\
Y_{E 8}=\frac{c}{2 \tan 22.5^{\circ}}
\end{array}\right.
\end{array}\right.
\end{aligned}
$$

A hexagon on the upper chord of the mid-span joint is created according to the above method, and connected with the joint of the three-direction grid type single-layer grid structure to form a partial web member of the three-direction grid type prestressed giant grid structure. The schematic diagram is as shown in Figure 11, and the length is marked as $L 4$. The length of L4 can be calculated by Formula (18):

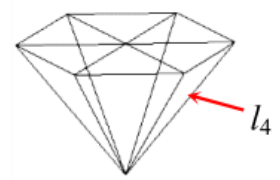

Fig. 11 - Connection between hexagon center and lower chord joints

$$
l_{4}=\sqrt{\left(\frac{\sqrt{3}}{2} c\right)^{2}+h^{2}}
$$

According to the local coordinate formula of the octagon, mutually crossing and connection with the upper part of the inverted quadrangular pyramid truss structure on the outer edge, so that 
the upper part of the structure forms four complete new out edges, namely the edge of the outermost edge of an upper chord of the whole structure, if it is external, cut it out.

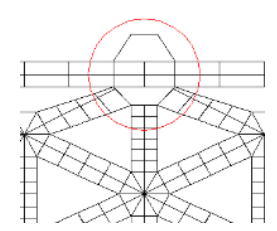

Fig. 12- Before octagon cutout

Each corner point of the polygon after cutting is respectively connected with the joint of the three-direction grid type single-layer grid structure to form the web member of the three-direction grid type prestressed giant grid structure, and the joint connection schematic diagram is shown in Figure 13 below.

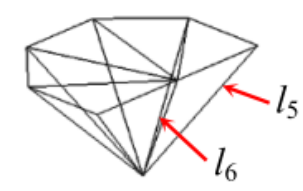

Fig. 13- Connection between octagon center and lower chord joints

The web member shown in the above figure is divided into two parts, one part is the corner point of the regular octagon connected with the three-direction grid type prestressed huge grid structure, and the length is marked as 15 , the other part is the center point of the regular octagon connected with the joint of the three-direction grid type prestressed huge grid structure, and the length marked as 16 can be calculated by formula (19):

$$
\left\{\begin{array}{l}
l_{5}=\frac{c}{2 \sin 22.5^{\circ}} \\
l_{6}=h
\end{array}\right.
$$

After the webs are formed, the center points of the hexagons and the octagons are connected with the corner points of the hexagons and the octagons to form partial top chords. Thus, a three-direction grid type prestressed mega-grid structure is finally formed.

\section{ANALYSIS OF DYNAMIC BEHAVIOR OF PRESTRESSED MEGA-RETICULATED STRUCTURE WITH 3-D LATTICE}

\section{Selection of structural calculation model}

\section{Calculation Model of Three-direction Grid Prestressed Reticulated Mega-structure}

Determination of basic geometry size: This paper refers to the geometry size design of $306 \mathrm{~m} \times 90 \mathrm{~m}$ hangar roof structure of Capital Airport [48], the control parameter of three-direction grid prestressed reticulated megastructure is set as follows: length $300 \mathrm{~m}$, span $150 \mathrm{~m}$, structural 
whole thickness $6 \mathrm{~m}$, structural megagrid number in long direction 4, structural megagrid number in span direction 4. The number of interjoint grids of long direction giant component is 10 , and the number of interjoint grids of span direction giant component is 8 . The number of interjoint grids of oblique giant components is 6 . Replace the lower chords on the main diagonal and the middle span with prestressed cables, and finally form a three-direction grid type prestressed mega-grid structure as shown in Figure 14.

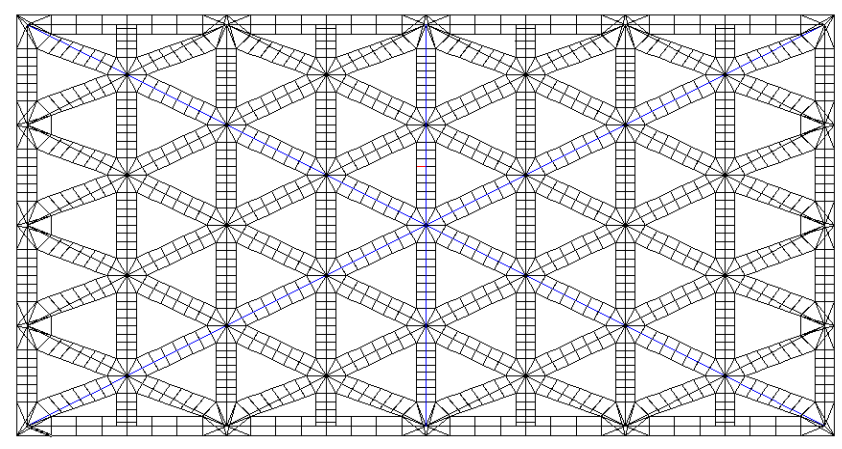

Fig. 14 - Arrangement position of prestressing cables

\section{Orthogonal Pyramid Grid Structure}

The modeling of square pyramid space truss structure can be obtained by inputting the corresponding parameters in MSTCAD software; The plane dimensions of square pyramid space grid structure are $300 \mathrm{~m}$ in length direction, $150 \mathrm{~m}$ in span direction, 31 grids in length direction, 19 grids in span direction and $6 \mathrm{~m}$ in thickness. The load and constraint conditions are the same as those of the prestressed mega grid structure. The established model is shown in Figure 16 below:

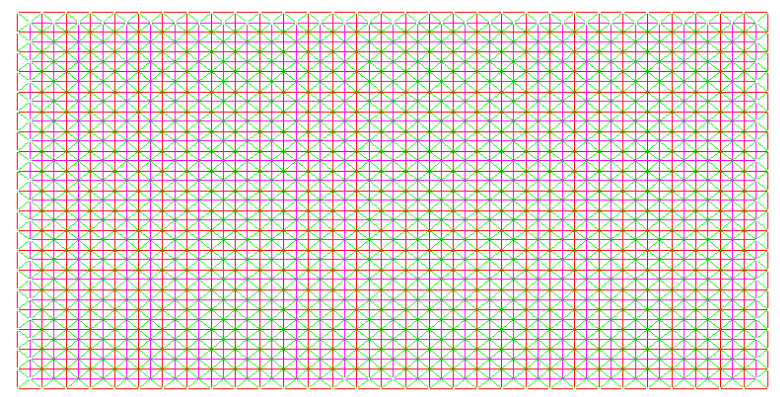

Fig. 15 - Orthogonal Pyramid Grid Structure

\section{Analysis of natural vibration characteristics of three-direction grid prestressed reticulated mega-structure}

The steel grade of the members is Q345, the nominal diameter of the prestressed cable is $300 \mathrm{~mm}$, and the basic level of the selected pretension is $23000 \mathrm{kN}$. The modal analysis of the three-direction grid type prestressed huge grid structure model is carried out by using sap2000 software, and 1.0 dead load +0.5 times live load is considered as the representative value of gravity load. In order to compare and analyze the dynamic characteristics of two kinds of space truss structures, the orthogonal square pyramid space truss in chapter 4 and chapter 3 are used to 
compare and analyze the dynamic characteristics of the two kinds of space trusses. Table 1 is the first 30 natural frequencies of square pyramid space grid structure, and Table 2 is the first 30 natural frequencies of three-direction grid prestressed reticulated mega-structure.

Tab. 1 - The first 30 natural frequencies $(\mathrm{Hz})$ of square pyramid space grid structures

\begin{tabular}{|c|c|c|c|c|c|c|c|c|c|c|}
\hline Order Number & 1 & 2 & 3 & 4 & 5 & 6 & 7 & 8 & 9 & 10 \\
\hline Natural Frequency & 0.38 & 0.44 & 0.595 & 0.73 & 0.84 & 1.04 & 1.12 & 1.29 & 1.38 & 1.45 \\
\hline Order Number & 11 & 12 & 13 & 14 & 15 & 16 & 17 & 18 & 19 & 20 \\
\hline Natural Frequency & 8.45 & 8.86 & 9.12 & 9.65 & 11.45 & 12.04 & 12.48 & 12.56 & 12.7 & 12.79 \\
\hline Order Number & 21 & 22 & 23 & 24 & 25 & 26 & 27 & 28 & 29 & 30 \\
\hline Natural Frequency & 12.8 & 13.02 & 13.67 & 14.21 & 14.45 & 14.54 & 15.64 & 15.87 & 16.08 & 16.08 \\
\hline
\end{tabular}

Tab. -: The first 30 natural frequencies $(\mathrm{Hz})$ of three-direction grid prestressed reticulated megastructure

\begin{tabular}{|c|c|c|c|c|c|c|c|c|c|c|}
\hline Order Number & 1 & 2 & 3 & 4 & 5 & 6 & 7 & 8 & 9 & 10 \\
\hline Natural Frequency & 1.38 & 2.44 & 2.59 & 3.73 & 3.83 & 4.03 & 5.12 & 6.29 & 7.38 & 7.45 \\
\hline Order Number & 11 & 12 & 13 & 14 & 15 & 16 & 17 & 18 & 19 & 20 \\
\hline Natural Frequency & 8.54 & 8.64 & 8.67 & 8.69 & 9.71 & 11.72 & 11.78 & 11.80 & 12.87 & 12.89 \\
\hline Order Number & 21 & 22 & 23 & 24 & 25 & 26 & 27 & 28 & 29 & 30 \\
\hline Natural Frequency & 12.9 & 12.97 & 13.05 & 13.12 & 13.22 & 14.42 & 15.54 & 15.64 & 16.73 & 16.76 \\
\hline
\end{tabular}




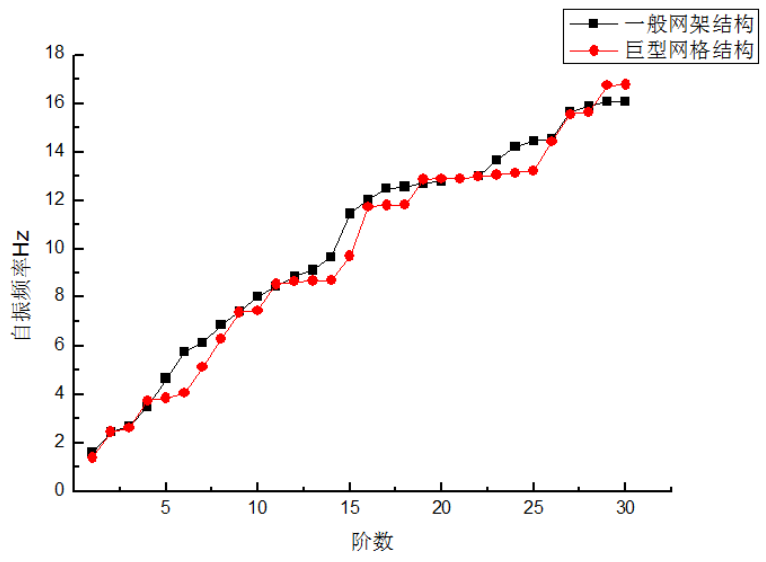

Fig. 16 - Comparison of the first 30 natural frequencies of the two structures

From Figure 16, it can be found that the natural frequency of the three-direction grid prestressed mega-grid structure is lower than that of the square pyramid grid structure, and its basic period is larger than that of the grid structure. The main reason is due to the three-direction grid type prestressed reticulated mega-structure caused by the intmemberuction of cable, with cable element as the tension element. It can also be concluded that the high order modes cannot be ignored in the dynamic response of the structure for the large span structure.

\section{CONCLUSION}

1. In this paper, the inverted pyramid truss is selected as the lattice mega-component of the three-direction grid prestressed reticulated mega-structure. By analyzing the connection form of the three-direction grid prestressed reticulated mega-structure, the specific ideas of "hexagon" and "octagon" cross connection composite joints are proposed, and the specific scheme of forming part of web members according to composite joints is proposed.

2. This paper determines the important control parameters of three-direction grid type prestressed reticulated mega-structure, and demonstrates the establishment of composite joints in the span and side composite joints, and finally chooses regular polygon to ensure that the mega-grid layout is uniform and the overall structure is reasonable.

3. According to the construction idea of three-direction grid type prestressed reticulated mega-structure, the hexagonal and octagonal joint coordinate schemes are deduced and formed, the geometric model establishment method of the hexagonal cross joint and the octagonal cross joint is proposed, and finally the specific scheme of threedirection grid type prestressed reticulated mega-structure geometric model construction is formed.

4. The quadrangular pyramid space grid structure with the same length, span, rise and grid size and the three-direction grid prestressed grid structure are calculated, and the 
advantages of three-direction grid prestressed grid structure in dynamic performance are compared and analyzed.

\section{REFERENCES}

[1] He Y., 2000. Shape analysis and mechanical model of reticulated mega-structure. In: Proceedings of the 9th Symposium on Spatial Structures, edited by China Civil Engineering Society, 6

[2] He Y., 2001. Study on the Shape, Static and Stability of Reticulated Mega-structure. Master's thesis, Zhejiang University, Hangzhou

[3] He Y., Li J., Zhou X., 2007. Composition, supporting method and mechanical model of spherical reticulated mega-structure. Journal of Hunan University (Natural Science Edition), vol 3: 11-14

[4] Bai Z. X., Liu X. L., Li Y. S., 2001. A NewType of Spatial Structure-Beam String structure. Spatial Structures, vol. 7: 33-38

[5] Zhang Y. G., 2000. Development of Cable-supported Structures in the Last Ten Years(1)-Concept of Cable-supported Strctures and Development of Plane Cable-supported Structures. Industrial Construction, vol. 2:105-113

[6] Saitoh M., Okada A., 1999. The Role of String in Hy-brid String Structure. Engineering Structures, vol. 21: 756-769. https://doi.org/10.1016/S0141-0296(98)00029-7

[7] Ding L. R., 2012. Analysis of static and dynamic characteristics of ribbed giant grid suspen-dome structure. Master's thesis, Xi'an University of Architecture and Technology, Xi'an

[8] Hu D., 2015. Study on static and stability of honeycomb reticulated spherical huge reticulated shell. Master's thesis, Hunan University, Hunan.

[9] Yao L., 2015. Study on static and stability of $800 \mathrm{~m}$ super-long Kiewitt reticulated mega-structure. Master's thesis, Harbin Institute of Technology, Harbin

[10] He Y. J., Zhou X. H., 2007. Static properties and stability of cylindrical ILTDBS reticulated megastructure with double-layer grid substructures. Journal of Constructional Steel Research, Vol. 63: 1580-1589. https://doi.org/10.1016/j.jcsr.2007.02.002

[11] He Y. J., Zhou X. H., Xu L., 2009. Stability of a cylindrical ILTDBS reticulated mega-structure with single layer LICS substructures. Journal of Constructional Steel Research, Vol. 65: 159-168.

https://doi.org/10.1016/j.jcsr.2008.05.013

[12] He Y. J., Zhou X. H., Xu L., 2009. Formation and stability of a cylindrical ILTDBS Reticulated megastructure braced with single-layer latticed membranous shell substructures. Thin-Walled Structures, Vol. 47: 537-546. https://doi.org/10.1016/j.tws.2008.10.001 